\title{
1 People with nicotine use-disorder exhibit more prefrontal 2 activity during preparatory control but reduced anterior 3 cingulate activity during reactive control
}

4

5 Shivam Kalhan ${ }^{\mathrm{a}^{*}}$, Li Peng Evelyn Chen ${ }^{\mathrm{a}}$, Marta I. Garrido ${ }^{\mathrm{a}, \mathrm{b}^{* *}}$, Robert Hester $^{\text {*** }}$

6 a-School of Psychological Sciences, University of Melbourne, Melbourne, Victoria, Australia

$7 \quad b$-Australian Research Council Centre of Excellence for Integrative Brain Function, Australia

$8 \quad * *$ Shared senior authors

9 * Corresponding author at:

10 School of Psychological Sciences, University of Melbourne, Redmond Barry Building, Parkville

11 Campus, Melbourne, Victoria, Australia.

12 E-mail address:

13 shiv.kalhan@gmail.com (Shivam Kalhan) 


\section{Abstract:}

18 Reduced inhibitory control and a hypersensitivity to reward are key deficits in drug-dependents,

19 however, they tend to be studied in isolation. Here we seek to understand the neural processes

20 underlying control over reward and how this is different in people with a nicotine use disorder

21 (pNUD). A novel variant of the monetary incentive delay task was performed by pNUD $(n=20)$

22 and non-smokers $(\mathrm{n}=20)$, where we added a stop-signal component such that participants had to

23 inhibit prepotent responses to earn a larger monetary reward. Brain activity was recorded using

24 functional magnetic resonance imaging (fMRI). We estimated stop signal reaction times (SSRT),

25 an indicator of impulsivity, and correlated these with brain activity. Inhibitory accuracy scores did

26 not differ between the control group and pNUD. However, pNUD had slower SSRTs, suggesting

27 that they may find it harder to inhibit responses. Brain data revealed that pNUD had greater

inhibitions over reward. In contrast, non-smokers had greater reactive control associated with more

SSRTs are slower. Overall, whilst the inhibition accuracy scores were similar between groups,

34 The findings suggest that increasing preparatory control in pNUD may be one possible treatment target in order to increase inhibitory control over reward.

\section{Keywords:}

38 Anterior cingulate cortex; inhibitory control; nicotine use-disorder; prefrontal cortex; striatum 
Addiction is a complex disorder which includes multiple decision-making symptoms ${ }^{1}$. Key

41 deficits include hypersensitivity towards drug-related rewards ${ }^{2}$, including towards monetary

42 rewards ${ }^{3}$, and reduced inhibitory $\operatorname{control}^{4}$. These deficits may contribute to the high persistence in

43 drug-seeking behaviors and impulsive decision-making found in people with a substance use

44 disorder (pSUD). Impulsivity is defined here as overvaluing a smaller immediate drug-reward

45 compared to the larger later reward of better health from abstinence ${ }^{5}$. A better understanding of

46 these processes may offer insight into ways of decreasing drug-seeking behaviors and avoiding

47 relapse by exercising control over immediate rewards. These differential processes of inhibiting a

48 response and the hypersensitivity to rewards have been relatively well studied; but are typically

49 studied independently.

The stop-signal task is commonly used to study processes involved in response inhibition ${ }^{6}$.

51 In this task, there is a prepotent tendency to respond to a target stimulus due to the frequent "go-

52 trials". However, less frequently, there is a stop-signal presented with a short latency to the target

53 stimulus, where individuals now need to withhold their response. These are termed "stop-trials".

54 The dorsolateral prefrontal cortex (dlPFC), including the inferior frontal gyrus (IFG) are associated

55 with successfully inhibiting a response during stop-trials ${ }^{7}$, both, prior to inhibition (preparatory

56 control $^{8,9}$ ) and during inhibition (reactive control ${ }^{10}$ ). Those with a SUD, including to nicotine, have

57 demonstrated reduced inhibitory control in this stop-signal task ${ }^{11-13}$ and this has been associated

58 with hypoactivity in regions within the $\mathrm{PFC}^{14}$.

The mechanisms underlying reward-processing have been studied through various

60 paradigms, including the monetary incentive delay (MID) task ${ }^{15}$. The MID task cues participants

61 on possible upcoming rewards and then asks them to respond to a target stimulus to win or lose

62 rewards - depending on the variant of the task used. The striatum has typically been associated 
63 with reward processing ${ }^{16}$ and in this MID task, increased striatal activation has been associated 64 with reward anticipation ${ }^{17}$. Administering the MID task to participants with a SUD has identified aberrant striatal functioning (with increases and decreases relative to controls), particularly when they are asked to anticipate rewards. This aberrant striatal functioning is thought to contribute to

67 the heightened impulsivity and impaired reward processing ${ }^{18}$ and although the opponent-process

68 theory ${ }^{19}$ suggests that this striatal aberration is a possible predictor of developing addictive-like behaviors, evidence supporting this relationship is mixed ${ }^{20,21}$. Lastly, PFC hypoactivation during reward processing is also found in pSUD, implying that there may be reduced inhibitory control 71 over choices with immediate rewards ${ }^{22}$.

Taken together, the PFC appears to increase cognitive control and the striatum plays a role

73 in processing and anticipating rewards. Both of these brain regions exhibit aberrant functioning in

74 addiction and possibly contribute to the reduced inhibitory control over immediate reward which

75 may drive impulsive decision-making. However, the neural mechanisms underlying how the two

76 processes interact remains less clear. Namely, how does the brain exercise inhibitory control over

77 reward in pSUD? Is this through modulation of PFC activity, striatal activity, or both? We

78 hypothesized that reduced PFC activity may be associated with failed inhibitions, consistent with

79 its role in cognitive control. We further hypothesized that increased striatal activity may also be

80 associated with failed inhibitions, by possibly anticipating and over-valuing the smaller sooner

81 rewards.

To investigate this question, we used a novel variant of the MID task - termed monetary

83 incentive control task (MICT). Here, we added the stop-signal component to the MID task. We 84 also used real monetary reward components of two modalities; smaller sooner and larger later. To 85 win the smaller reward, participants had to quickly respond to a target stimulus. To win the larger 
reward, participants had to successfully inhibit their response on most of the stop-trials. If

87 participants responded quickly on stop-trials - they still won the small reward. Previous studies ${ }^{23,24}$ have also combined the stop-signal task with a reward manipulation, however, a critical distinction is that in our task there is also a reward given for failing to inhibit, whereas reward was only given for successfully inhibiting in the previous tasks. Failed inhibition was rewarded to better simulate real-world abstinence where failing to inhibit comes with a small immediate reward. Importantly, participants were not updated on their progress towards winning the larger reward, since again, the aim was to simulate real world abstinence and attempting abstinence does not come with the immediate certainty of the receipt of a larger reward (better health in the future). However, a relapse comes with the more certain small reward (drug). the stop-signal task by increasing engagement of control-related PFC activity ${ }^{25}, 2$ ) reduce conflict during a conflict-response task, associated with increased functional connectivity between intraparietal sulcus and the striatum, engaging more top-down control $^{26}$ and, 3) increase performance in the Stroop-task in both healthy controls (by enhancing dorsolateral PFC activity) and in people with a cocaine use-disorder (by enhancing occipital lobe activity and the functional connectivity between the dorsolateral PFC and striatum $)^{27}$. In contrast, our task provides a smaller sooner incentive for failed inhibitions, and accordingly, we hypothesized that this may make inhibitions more difficult by engaging striatal anticipatory activity, particularly in pSUD, and 105 successful inhibitions may require increased PFC engagement, especially to exercise control over the smaller sooner incentive for failed inhibitions. There was also a manipulation where 107 participants were cued on the probability that the upcoming trial would be a 'stop' trial. This manipulation was used to investigate the potential interaction between reward anticipation and 
109 'stop' difficulty, with a higher probability condition hypothesized to engage control related PFC

110 regions and a higher stop accuracy score.

111 We recorded brain activity using functional magnetic resonance imaging (fMRI) while

112 participants performed this task. Both groups of people with a nicotine use disorder (pNUD) and

113 non-smokers healthy controls underwent this task. Nicotine withdrawal can produce neurotoxic

114 effects in the mesolimbic reward systems comparable to other drugs of abuse including

115 amphetamine, cocaine and opiates ${ }^{28}$. We also estimated stop signal reaction times (SSRTs), which

116 is a measure of how effortful it is to inhibit a response, as well as an index of impulsivity ${ }^{29}$. Overall,

117 we investigated how the brain may exercise control over smaller sooner rewards to attain a larger

118 later reward in pNUD and non-smokers. 


\section{MATERIALS AND METHODS}

122

124

125

126

127

128

129

130

131

132

133

134

135

136

\section{Participants}

Participants were recruited through advertisements at the University of Melbourne, and through a community website. All participants provided written informed consent which was approved by Human Ethics Committee of the University of Melbourne and the Royal Children's Hospital. The pNUD group consisted of twenty individuals (10 males, 10 females; mean age $=$ 24.3 years, standard deviation $=4.7$, range $=18-34)$. The brain structural images for one pNUD group participant was not retrievable, hence brain imaging data for this participant was not analyzed. The control group consisted of twenty non-smokers (10 males, 10 females; mean age $=$ 23.7 years, standard deviation $=4.3$, range $=18-32$ ). One participant in the control group had an anatomical anomaly, however, this was very minor, and the participant was included in the analysis. All participants were right handed - determined by the Edinburgh Handedness Inventory 30. Participants in the control group had smoked less than 6 cigarettes in their lifetime. Participants in the pNUD group smoked at least fifteen cigarettes daily and the average Fagerstrom Test for Nicotine Dependence (FTND) score was 3.95, indicating close to moderate dependence ${ }^{31}$. The group average for years of cigarettes smoked was 7.6 years.

Prior to the experiment, the pNUD group had been abstinent for at least three hours. This was confirmed by both a self-report and the carbon monoxide breath measure. Exclusion criteria for both groups consisted of a history of neurological or psychiatric disorder, current use of psychotropic medication (other than nicotine for the pNUD group). Scanning data was collected between 9am and 5pm across participants, and they were advised not binge prior to abstaining for the 3-hours. Participants also arrived at least 1-hour prior to scanning for task preparation/practice, which also prevented them from smoking. The 3-hour abstinence window was chosen due to 
144 nicotine's half-life of approximately 2-hours ${ }^{32}$, and data suggesting that 3-hours abstinence did not

145 produce withdrawal effects on cognition ${ }^{13}$. was a variable delay presented for $2-4 \mathrm{~s}$, with $1 \mathrm{~s}$ jitter. This delay period was termed the either a "X" or an "O", presented for $400 \mathrm{~ms}$, with a blank screen for $600 \mathrm{~ms}$ following this.

Participants had to press the correct button (left or right) associated with the target letter within $400 \mathrm{~ms}$ to get the small $20 \varnothing$ reward, if this was a reward trial. For stop trials, a square border around the target letter would appear after $150 \mathrm{~ms}$ latency. If this stop trial was a reward trial and participants withheld their response at $60 \%$ or more of these rewarding stop trials, they would get the large \$20 reward at the end of the task. If they responded within 400ms to the rewarding stop trial - they would still get the small $20 \varnothing$ reward. For neutral trials - no monetary reward could be received, irrespective of performance. Overall, there were four conditions based on the following trial types - 1) reward trial with $20 \%$ probability $(\mathrm{R} 20), 2)$ neutral trial with $20 \%$ probability (N20), could all be either "go" or "stop" trial types. Following the trial - participants were presented with feedback for $1.5 \mathrm{~s}$. The feedback indicated their performance and if they had won the small $20 \varnothing$ 
167 how much money they had earned from go-trials. However, importantly, feedback on stop trial accuracy and any associated monetary gain on the larger $\$ 20$ reward was not provided until the end of the task.

171 break-up of 'stop' and 'go' trials (25\% stop-trials and $75 \%$ go-trials) is commonly used to create

172 the prepotent tendency to respond in the stop-signal paradigm ${ }^{6}$. The task was split into six runs,

173 with 36 trials per run. Trials were presented in a pseudo-random, intermixed design, within each

174 run. The R20 condition had a total of 78 go-trials and 18 stop-trials, R40 had 30 go-trials and 18

175 stop-trials, N20 39 go-trials and 9 stop-trials, N40 had 15 go-trials and 9 stop-trials. The task lasted 176 approximately 45 minutes including rest breaks between runs. Please see supplementary material 177 methods for 1) the apparatus details, 2) experimental procedure, 3) MRI sequences used and 4) the 178 methods of SSRT estimation.

For performance indices we used the stop-accuracy percentage (calculated as the number of stop-trials where participants inhibited their responses divided by the total number of stop-trials) and go-accuracy percentages (calculated as the number of responses made on go-trials in under 400ms divided by the total number of go-trials). Data above and/or below three standard deviations

184 from the mean were removed as outliers. To test for significance, we used repeated-measures analysis of variance (ANOVA). There were three factors and each factor had two levels $(2 \times 2 \times 2$ ANOVA design). These included; 1) factor of probability, with levels of low (20\%) and high 187 (40\%) probabilities, 2) Factor of reward, with levels of rewarding trial and neutral trial, and 3) 188 factor of group, with levels of pNUD group and control group. To test for simple effects, 189 independent t-tests were conducted where ANOVA yielded significant results, corrected for 
190

191

192

194

195

196

197

multiple comparisons using Šidák correction. The partial eta-squared $\left(\eta_{\mathrm{p}}{ }^{2}\right)$ was calculated as a measure of effect size with 0.01 being small, 0.06 being medium and 0.14 being large.

fMRI Analysis

Images were pre-processed using SPM default functions with steps in the order of realignment, co-registration, segmentation and normalized into MNI-space. The images were smoothed using the 6mm FWHM Gaussian kernels. Runs with movement of greater than 4mm (one voxel size) in either of the $\mathrm{x}, \mathrm{y}, \mathrm{z}$ planes were removed from further analysis. All general linear models (GLMs) consisted of six movement regressors. A high-pass filter of 128s was used.

inhibitions. This gave eight regressors of interest in our first-level GLM (four conditions for failed and successful inhibitions). These were all analyzed for four different epochs; 1) cue epoch, 2) anticipation epoch, 3) trial epoch (from onset of stop signal) and 4) feedback epoch. We modelled cue and anticipation epochs separately to investigate any possible brain activity differences during encoding of the cues, which are only presented during the cue epoch and not during the anticipation epoch. All task epochs apart from the epoch of interest was modelled as a regressor of no interest. For example, when analyzing for effects at the stop trials for the cue epoch - all other epochs at these stop trials were modelled as regressors of no interest, in addition to also all epochs in gotrials. This was done to reduce noise at our epoch and trial of interest, while reducing possible confounds from other trials and epochs. Lastly, for the feedback epoch, failed inhibitions where a 20c reward was won, was excluded. This was to remove any potential confound of the small 20c reward and to directly compare feedback of 1) "correct 0c" for successful inhibitions and "miss 0c" for failed inhibitions. 
213 included three factors, with two levels each; 1) group (control and pNUD), 2) inhibition accuracy

214 (failed and successful) and 3) reward (neutral trial or reward trial). Main effects and interactions

215 were tested and, t-contrasts to examine condition-specific results. For model-based results, SSRTs

216 for each participant, across each condition were aligned and used as covariates of interests at the

217 second-level. This GLM consisted of cells for each reward and probability condition, for each of

218 the two groups. There was no inhibition accuracy factor in this GLM as SSRT is not estimated for

219 failed inhibitions (where a response is made). All data presented has threshold of $\mathrm{p}<0.05$ family-

220 wise error corrected (FWE) at the cluster-level, unless specified otherwise. Background brain

221 image used for figures is from SPM canonical, which is an average T1 from 305 individuals, in

222 MNI-space.

223 RESULTS

224 Behavior

225

pNUD have similar stop accuracy scores to controls

As in Figure 3a, there were no significant differences in stop accuracy scores between 227 groups (main effect of group: $\mathrm{F}(1,38)=3.2, \mathrm{p}=0.08, \eta_{\mathrm{p}}^{2}=0.078$ ), and no main effect of reward

$228\left(\mathrm{~F}(1,38)=2.3, \mathrm{p}=0.13, \eta_{\mathrm{p}}^{2}=0.058\right)$. There was a main effect of probability $(\mathrm{F}(1,38)=65.95, \mathrm{p}<$ $\left.2290.0001, \eta_{\mathrm{p}}^{2}=0.63\right)$, with performance better in the $40 \%$ probability condition compared to the $23020 \%$. There was also a significant group $x$ probability interaction $\left(F(1,38)=4.70 \mathrm{p}=0.036, \eta_{\mathrm{p}}{ }^{2}=\right.$

231 0.11), which appeared to be driven by the differences between pNUD and control groups in the $232 \mathrm{~N} 20$ condition ( $\mathrm{t}$-test, $\mathrm{p}=0.007$ ). Twelve participants from the pNUD group won the larger later $233 \$ 20$ reward, and thirteen participants from the control group. This was won by successfully 
234 inhibiting a response on $60 \%$ or more of the reward stop-trials. Overall, the stop-accuracy scores

235 suggest that both groups perform better when cued that the upcoming trial has a higher probability

236 of being a stop-trial, and this is irrespective of whether this is a reward or a neutral trial. See Figure

237 S1 (supplementary materials) for accuracy scores and reaction times for go-trials.

Figure 3b shows SSRT estimates where pNUD had a slower SSRT compared to controls (main effect of group: $\mathrm{F}(1,37)=8.09, \mathrm{p}<0.01, \eta_{\mathrm{p}}^{2}=0.18$ ). While pNUD had a similar stopaccuracy score, the slower SSRT suggest the pNUD group found inhibiting a response more

242 effortful than controls. Independent t-tests indicated the pNUD group had a significantly slower

243 SSRT for the N20 ( $p=0.0054)$ and R40 ( $p=0.034)$. There were no significant differences in the $244 \mathrm{~N} 40(\mathrm{p}=0.25)$ and $\mathrm{R} 20(\mathrm{p}=0.097)$ conditions. We found a main effect of probability $(\mathrm{F}(1,37)=$

$\left.24513.13, \mathrm{p}<0.001, \eta_{\mathrm{p}}^{2}=0.26\right)$ where the $40 \%$ condition had faster SSRTs. We also found a main 246 effect of reward $\left(\mathrm{F}(1,37)=12.96, \mathrm{p}<0.001, \eta_{\mathrm{p}}{ }^{2}=0.26\right)$ where reward trials had faster SSRTs.

247 Lastly, there was no significant group and reward interaction $\left(\mathrm{F}(1,37)=1.1, \mathrm{p}=0.3, \eta_{\mathrm{p}}^{2}=0.03\right)$.

248 Overall, we find that the pNUD group is more 'impulsive' in their responding based on their slower 249 SSRTs.

\section{Brain fMRI Activity}

254 neutral (Figure 4a). Failed inhibitions have greater precentral and posterior medial frontal cortex

255 (pmFC) activity (Figure 4b). The pNUD group exhibits more control related activity in the IFG, 
middle frontal gyrus (MFG) and superior frontal gyrus (SFG) compared to non-smokers, in reward trials which were successfully inhibited (Figure 4c). The pNUD group also has more activity in the pre and postcentral gyri prior to successful inhibitions in the reward trials. Lastly, there was more activity in the fusiform gyrus for the $40 \%>20 \%$ probability contrast (figure not shown), which is consistent with the literature on the fusiform gyrus' activation during the stop signal task, playing a role in correctly recognizing cues and their salience in inhibiting a response ${ }^{33-35}$.

There is greater striatal and insula activity at the anticipation epoch for reward > neutral trials (Figure 5a). Figure 5b shows activity in angular gyrus prior to successful inhibitions,

264 consistent with previous literature ${ }^{9}$. Failed inhibitions (Figure 5c) have more activity in the pre 265 and postcentral gyri. There is also more activity in the medial cortical regions (anterior cingulate cortex; ACC, mid cingulate cortex; MCC and posterior medial frontal cortex; pmFC) associated

267 with these failed inhibitions. The pNUD group in the anticipation epoch exhibits more control 268 related activity in the IFG and MFG, as well as pre and postcentral activity (Figure 5d). 269 Interestingly, pNUD in this epoch have more IFG activity even prior to failed inhibitions in the 270 neutral trials (Figure 5e). See Table S1 and S2 in supplementary materials for a full list of brain 271 regions activated in these epochs and their respective MNI coordinates and individual group 272 activities).

Figure $6 \mathrm{a}$ shows the components of reactive control, where participants inhibit the 276 prepotent response after seeing the stop signal. These successful inhibitions were associated with 277 greater activity in control related regions (ACC, SFG, MFG), as well as the angular gyrus (Figure 
6a). Successfully inhibiting reward trials engages more IFG, insula and striatum compared to successfully inhibiting neutral trials (Figure 6b). Non-smokers have greater ACC activation compared to smokers (Figure 6c), and this may suggest that non-smokers have more reactive control. See Table S3 in supplementary materials for a full list of brain regions activated in this epoch and their respective MNI coordinates and individual group activities.

\section{Feedback Epoch}

Brain activity when processing feedback of "correct 0c" after successful inhibitions, contrasted with "miss 0c" after an incorrect response is shown in Figure 7a. Interestingly, there is striatal activity after successful inhibitions. Following successful inhibitions, there was also more activity in the ACC, SFG and MFG. In contrast, there was more insula and pmFC activity following failed inhibitions (Figure $7 b$ ). The pNUD group exhibited greater pmFC and SFG activity following failed inhibitions in neutral trials compared to controls (Figure 7c). In contrast, after successful inhibitions for reward trials, the pNUD group had greater activity in the medial cortical regions (cingulate gyrus, SFG, pmFC) and the IFG. See Table S4 in supplementary materials for a fill list of brain regions activated in this epoch and their respective MNI coordinates and individual group activities.

\section{Model-based SSRT brain activity correlations}

The SSRT estimates of each participant across all conditions, between both groups, were used as covariates at the second level analysis (Figure 8). Across all epochs, we found that with slower SSRTs in the pNUD group, there is more activity within the PFC and parietal regions. 
For the control group, we did not see SSRT brain correlations except for in the feedback

301

302

303

304

305

306

307

308

309

310

311

312

313

314

315

316

317

318

319

320

321

322

epoch where a slower SSRT correlates with greater activity in the pmFC. See Table S5 in supplementary materials for a full list of brain regions activated in this epoch and their respective MNI coordinates.

\section{DISCUSSION}

Here we investigated the underlying brain processes involved in exerting control over rewards in pNUD and healthy controls. Overall, we found that pNUD do not differ from controls in their stop-accuracy scores (Figure 3a). However, they had slower SSRTs (Figure 3b). The combination of these findings suggests that pNUD require more effort and/or have a greater difficulty inhibiting their prepotent 'go-processes'. This is consistent with previous findings where slower SSRTs were found in pSUD, including to nicotine ${ }^{12,13}$ and a slower SSRT predicts a higher dependence to nicotine ${ }^{36}$. There was also a significant difference in the stop-accuracy score between groups specifically in the N20 condition, where the pNUD group had lower stop-accuracy scores. The difference here may be due to the task design where neutral and reward conditions are interleaved. Another speculative possibility is that the dependant group may prioritise their limited cognitive resources on inhibiting in other conditions, since these other conditions may either lead to a reward (for R20 and R40 conditions) or have a higher chance of being a stop trial (N40 condition). Overall, the pNUD group's response inhibition performance was comparable to controls, but achieving parity required more cognitive effort.

The pNUD group did have greater activity in their cognitive control related prefrontal regions (IFG and MFG) prior to successful inhibitions for reward trials. This was during both, the cue and anticipation epochs. The increased activity might suggest the pNUD group engages in 
more preparatory control than healthy controls to achieve similar stop accuracies. Indeed, greater preparatory activity in prefrontal regions have previously been shown to aid in inhibitory processes $^{8,9}$. Further, increased IFG activation for more difficult stops were previously reported ${ }^{37}$. The hypothesis that increased IFG activity in pNUD indicates more effort in stopping, is consistent with our SSRT results. This finding of increased preparatory control in the pNUD group is contrary to another recent finding, in people with a cocaine use disorder, found to have reduced PFC related preparatory contol ${ }^{23}$. One explanation for the contrasting results may be due to the differences in the severity of dependence, where our sample had close to moderate levels and therefore may have a greater inhibitory control capacity relative to people with a cocaine use disorder. A second possibility is our short 3-hour abstinence window, which may still produce some stimulant-related effects of nicotine and possibly cotinine (a metabolite of nicotine with a much longer half-life ${ }^{38}$ ) on cognition and may therefore facilitate this "adaptive" pattern of behavior where reduced reactive control is compensated for by increased preparatory control. Interestingly, the pNUD group also exhibits greater activity in the pre and postcentral gyri, prior to successful inhibitions in reward trials. The increased precentral motor-related activity may be inhibited by the increased prefrontal control activity, contributing to the successful stop. Another possibility is that the precentral activation may be playing a role in motor inhibition as supported by findings from $\mathrm{Li}$, Huang, Constable, \& Sinha (2006), where precentral activation correlated with smaller SSRTs (or efficient inhibitions).

The pNUD in the anticipation epoch had more IFG activity prior to failed inhibitions in neutral trials (Figure 5e). Increased IFG activity prior to failed inhibitions observed here suggests that greater preparatory IFG activity does not guarantee a successful inhibition and may also need engagement from other control related prefrontal regions, such as MFG, as is the case prior to the 
successful inhibitions (Figure 5d). The finding that IFG may not guarantee a successful inhibition inhibitions in reward trials, aiding them in achieving similar stop accuracy scores to the controls, albeit with slower SSRTs. During the trial epoch, when the stop signal is detected, we see components of reactive control over reward. Successful inhibitions, across both groups, engaged more prefrontal control regions (MFG, SFG) and the ACC. These regions may aid in successfully inhibiting the go-processes and therefore contribute to the successful stop. The ACC has been suggested to play a more complicated and non-specific role in the stop signal task ${ }^{39}$, however, one

356 of its key roles has been implicated in inhibiting responses ${ }^{41}$. We therefore interpret ACC activity

357 here as playing a role in facilitating response inhibition. Interestingly, the control group had greater 358 ACC activity compared to the pNUD group during successful inhibitions in reward trials, suggesting that while the pNUD group may have an increased preparatory control, the control group have greater reactive control. The combination of these findings suggests that greater preparatory control (with increased IFG and MFG activity) reduces the need for high levels of reactive control to inhibit a prepotent response; as is the case for the dependent group. The control

363 group, on the other hand, show less preparatory control but increased reactive control (with 364 increased ACC activity) and can still reliably inhibit their responses. The control group may 365 therefore find it less difficult to inhibit their responses and may not require the upregulated preparatory control for a successful stop. Our SSRT results showing the control group have shorter SSRTs is consistent with this interpretation in that they may find it less difficult to inhibit responses 
control. Overall, both groups have similar inhibition accuracy, but achieve this through different processes. At the feedback epoch, there was increased striatal activation following successful

371 inhibitions when contrasted with failed inhibitions, in both groups. Given the striatum's role in

372 reward processing ${ }^{16}$, striatal activity here is consistent with participants anticipating the larger $\$ 20$

373 reward and this may play a role in motivating further response inhibitions to obtain the larger later

374 reward. Further, the feedback of "correct 0c" contrasted with "miss 0c" may also exhibit a 375 component of intrinsic reward processing for correctly performing the task. Contrary to our 376 hypothesis, we did not see striatal activity during impulsive responses (for the smaller 20c reward),

377 as previous studies have found ${ }^{17}$. Instead, the striatum was engaged during successful inhibitions

378 in reward trials over neutral (Figure 6b). This may be due to the participants' goal of attaining the

379 larger later reward as compared to the smaller sooner, where progress towards the larger later 380 reward engaged striatal anticipatory activity. Therefore, the small 20c "reward" may be considered 381 neutral or even punishing by the participants given the context of the trials, and the overall goal of 382 attaining the larger later reward.

Following successful inhibitions (in the feedback epoch), there was also more activity in

384 the ACC, SFG and MFG. These brain regions have previously been implicated in processing 385 feedback, including positive feedback ${ }^{42,43}$. Interestingly, the pNUD group had greater activity in 386 the medial cortical regions and the IFG, following successful inhibitions in reward trials, compared 387 to controls. Feedback processing and associated activity in these medial cortical regions have 388 previously been shown to increase task performance ${ }^{43}$. One interpretation may be that increased 389 activation in these regions for the pNUD group aids in improving stop accuracy scores to match 390 those of the control group. Decreases in inhibitory control may lead to different compensatory 
neural strategies by the pNUD group and increasing feedback processing during successful inhibitions may therefore be one such compensatory neural strategy.

The model-based SSRT brain correlations showed that the pNUD group has more cognitive control related activity correlating with slow SSRTs, across all epochs. On the contrary, the control group shows more control related SFG and precentral activity to correlate with fast SSRTs, although only at a relaxed statistical threshold (SFG; $p=0.066$ FWE (cluster-level) and precentral; $\mathrm{p}=0.057 \mathrm{FWE}$ (cluster-level)). Overall, one may expect greater engagement of these control related regions to faster SSRTs, hence enabling more efficient stopping, as found by Galván, Poldrack, Baker, McGlennen, \& London (2011), where both smokers and non-smokers had greater activity in control related regions that correlated with faster SSRT. However, Galván and colleagues (2011) did not find SSRT differences between groups. Other studies correlating SSRT scores with fMRI data have found that faster SSRTs correlate more strongly with control related regions (medial cortical regions, SFG) and motor regions such as the pre-supplementary motor area and precentral ${ }^{39,45,46}$. These were all investigated in healthy non-smoker participants. Consistent with these studies, we found more precentral and PFC activity for the control group. However, for the pNUD group it appears that slower SSRT engages more control related regions. One interpretation to bring together these SSRT results across groups may be that the SSRTs reflect the cognitive effort required for stopping, or difficulty in inhibiting. For the control group, more effort is required for fast inhibitions, hence greater control related SFG activity. In contrast, the pNUD group appears to find the slower SSRTs more effortful, consistent with greater control related PFC activity.

In sum, we find that both control and pNUD participants exhibit similar stop accuracy scores. However, the brain processes exhibited to achieve this are different between the two 
414 groups. The pNUD group shows greater inhibitory control related activity prior to successful

415 inhibitions over reward, whereas, the control group exhibit greater reactive control with greater

416 ACC activity during the inhibition epoch. Collectively, our results shed light on some of the brain

417 processes involved in successfully exhibiting control over immediate small rewards in favor of 418 greater delayed gratification in non-smokers and pNUD. 


\section{Acknowledgments}

The project was funded by the NHMRC project grant 1050766 to Robert Hester. Shivam Kalhan was supported by the Velma Stanley PhD Scholarship and by the Australian Government Research Training Program Scholarship, provided by the Australian Commonwealth Government and the University of Melbourne.

\section{Author Contributions}

All authors contributed to this paper. E.C collected the data and performed preliminary analysis. R.H designed the experiment and wrote the paper. M.I.G wrote the paper. S.K analyzed data and wrote the paper.

\section{Conflict of Interest}

None.

\section{Data A vailability Statement}

The data that support the findings of this study are available on request from the corresponding author. The data are not publicly available due to privacy or ethical restrictions. 


\section{References}

491

1. Redish AD, Jensen S, Johnson A. A unified framework for addiction: Vulnerabilities in the decision process. Behav Brain Sci. 2008;31(4):415. doi:10.1017/S0140525X0800472X

2. Koob GF, Volkow ND. Neurobiology of addiction: a neurocircuitry analysis. The Lancet Psychiatry. 2016;3(8). doi:10.1016/S2215-0366(16)00104-8

497

498

499

500

Kirby KN, Petry NM, Bickel WK. Heroin addicts have higher discount rates for delayed rewards than non-drug-using controls. J Exp Psychol Gen. 1999;128(1):78. doi:10.1037/0096-3445.128.1.78

4. Verdejo-Garcia A, Chong TTJ, Stout JC, Yücel M, London ED. Stages of dysfunctional decision-making in addiction. Pharmacol Biochem Behav. 2018;164:99-105. doi:10.1016/j.pbb.2017.02.003

5. Bickel WK, Yi R, Mueller ET, Jones BA, Christensen DR. The behavioral economics of drug dependence: Towards the consilience of economics and behavioral neuroscience. Curr Top Behav Neurosci. 2010:319-341.doi:10.1007/7854_2009_22

6. Logan GD, Cowan WB. On the ability to inhibit thought and action: A theory of an act of control. Psychol Rev. 1984;91(3):295. doi:10.1037/0033-295X.91.3.295

7. Aron AR, Poldrack RA. Cortical and subcortical contributions to stop signal response inhibition: Role of the subthalamic nucleus. J Neurosci. 2006;26(9):2424-2433. 
511 8. Chikazoe J, Jimura K, Hirose S, Yamashita KI, Miyashita Y, Konishi S. Preparation to inhibit a response complements response inhibition during performance of a stop-signal task. J Neurosci. 2009;29(50):15870-15877. doi:10.1523/JNEUROSCI.3645-09.2009

9. Hu S, Li CSR. Neural processes of preparatory control for stop signal inhibition. Hum Brain Mapp. 2012;33(12):2785-2796. doi:10.1002/hbm.21399

10. Boehler CN, Appelbaum LG, Krebs RM, Hopf JM, Woldorff MG. Pinning down response inhibition in the brain - Conjunction analyses of the Stop-signal task. Neuroimage. 2010;52(4):1621-1632. doi:10.1016/j.neuroimage.2010.04.276

11. Brevers D, Bechara A, Kilts CD, et al. Competing motivations: Proactive response inhibition toward addiction-related stimuli in quitting-motivated individuals. J Gambl Stud. 2018;34(3):785-806. doi:10.1007/s10899-017-9722-2 substance abuse and addiction: A meta-analysis. Drug Alcohol Depend. 2014;145:1-33.

525 13. Charles-Walsh K, Furlong L, Munro DG, Hester R. Inhibitory control dysfunction in nicotine dependence and the influence of short-term abstinence. Drug Alcohol Depend.

528 14. Feil J, Sheppard D, Fitzgerald PB, Yücel M, Lubman DI, Bradshaw JL. Addiction, compulsive drug seeking, and the role of frontostriatal mechanisms in regulating inhibitory control. Neurosci Biobehav Rev. 2010;35(2):248-275.

532 15. Knutson B, Westdorp A, Kaiser E, Hommer D. FMRI visualization of brain activity 
during a monetary incentive delay task. Neuroimage. 2000;12(1):20-27.

doi:10.1006/nimg.2000.0593

16. Delgado MR. Reward-related responses in the human striatum. Ann N Y Acad Sci. 2007;1104(1):70-88. doi:10.1196/annals.1390.002

17. Oldham S, Murawski C, Fornito A, Youssef G, Yücel M, Lorenzetti V. The anticipation and outcome phases of reward and loss processing: A neuroimaging meta-analysis of the monetary incentive delay task. Hum Brain Mapp. 2018;39(8):3398-3418.

doi:10.1002/hbm.24184

18. Balodis IM, Potenza MN. Anticipatory reward processing in addicted populations: A focus on the monetary incentive delay task. Biol Psychiatry. 2015;77(5):434-444. doi:10.1016/j.biopsych.2014.08.020

19. Solomon RL. The opponent-process theory of acquired motivation: The costs of pleasure and the benefits of pain. Am Psychol. 1980;35(8). doi:10.1037/0003-066X.35.8.691

20. Bjork JM. The Ups and Downs of Relating Nondrug Reward Activation to Substance Use Risk in Adolescents. Curr Addict Reports. 2020;7(3). doi:10.1007/s40429-020-00327-7

21. Sherman L, Steinberg L, Chein J. Connecting brain responsivity and real-world risk taking: Strengths and limitations of current methodological approaches. Dev Cogn Neurosci. 2018;33. doi:10.1016/j.den.2017.05.007

22. Goldstein RZ, Volkow ND. Dysfunction of the prefrontal cortex in addiction: Neuroimaging findings and clinical implications. Nat Rev Neurosci. 2011;12(11):652-669. doi:10.1038/nrn3119

23. Nigam KB, Straub LK, Zuniga EA, et al. Blunted prefrontal signature of proactive inhibitory control in cocaine use disorder. Drug Alcohol Depend. 2021;218. 
doi:10.1016/j.drugalcdep.2020.108402

557 24. Chikara RK, Chang EC, Lu YC, Lin DS, Lin CT, Ko LW. Monetary reward and punishment to response inhibition modulate activation and synchronization within the inhibitory brain network. Front Hum Neurosci. 2018;12. doi:10.3389/fnhum.2018.00027

25. Boehler CN, Schevernels H, Hopf JM, Stoppel CM, Krebs RM. Reward prospect rapidly speeds up response inhibition via reactive control. Cogn Affect Behav Neurosci. 2014;14(2). doi:10.3758/s13415-014-0251-5

563 26. Padmala S, Pessoa L. Reward reduces conflict by enhancing attentional control and biasing visual cortical processing. J Cogn Neurosci. 2011;23(11). doi:10.1162/jocn_a_00011

566 27. Rosell-Negre P, Bustamante JC, Fuentes-Claramonte P, Costumero V, Llopis-Llacer JJ, Barró S-Loscertales A. Reward contingencies improve goal-directed behavior by enhancing posterior brain attentional regions and increasing corticostriatal connectivity in cocaine addicts. PLoS One. 2016;11(12). doi:10.1371/journal.pone.0167400

28. Epping-Jordan MP, Watkins SS, Koob GF, Markou A. Dramatic decreases in brain reward

572 29. Verbruggen F, Logan GD. Models of response inhibition in the stop-signal and stop-

573 change paradigms. Neurosci Biobehav Rev. 2009;33(5):647-661.

$574 \quad$ doi:10.1016/j.neubiorev.2008.08.014

575 30. Oldfield RC. The assessment and analysis of handedness: The Edinburgh inventory. Neuropsychologia. 1971;9(1):97-113. doi:10.1016/0028-3932(71)90067-4

577 31. Heatherton TF, Kozlowski LT, Frecker3 RC, Fagerstrom^ ${ }^{\wedge}$ K-O. The Fagerstrom Test for 578 Nicotine Dependence: A Revision of the Fagerstrom Tolerance Questionnaire. Vol 86.; 
1991. https://onlinelibrary.wiley.com/doi/abs/10.1111/j.1360-0443.1991.tb01879.x.

580

581

582

583

584

585

586

587

588

589

590

591

592

593

594

595

596

597

598

599

600

601

Accessed May 17, 2021.

32. Benowitz NL, Jacob P, Jones RT, Rosenberg J. Interindividual variability in the metabolism and cardiovascular effects of nicotine in man. J Pharmacol Exp Ther. $1982 ; 221(2)$.

33. Pawliczek CM, Derntl B, Kellermann T, Kohn N, Gur RC, Habel U. Inhibitory control and trait aggression: Neural and behavioral insights using the emotional stop signal task. Neuroimage. 2013;79:264-274. doi:10.1016/j.neuroimage.2013.04.104

34. Fauth-Bühler M, de Rover M, Rubia K, et al. Brain networks subserving fixed versus performance-adjusted delay stop trials in a stop signal task. Behav Brain Res. 2012;235(1):89-97. doi:10.1016/j.bbr.2012.07.023

35. Simmonds DJ, Pekar JJ, Mostofsky SH. Meta-analysis of Go/No-go tasks demonstrating that fMRI activation associated with response inhibition is task-dependent. Neuropsychologia. 2008;46(1):224-232. doi:10.1016/j.neuropsychologia.2007.07.015

36. Billieux J, Gay P, Rochat L, Khazaal Y, Zullino D, Van der Linden M. Lack of inhibitory control predicts cigarette smoking dependence: Evidence from a non-deprived sample of light to moderate smokers. Drug Alcohol Depend. 2010;112(1-2):164-167. doi:10.1016/j.drugalcdep.2010.06.006

37. Hughes ME, Johnston PJ, Fulham WR, Budd TW, Michie PT. Stop-signal task difficulty and the right inferior frontal gyrus. Behav Brain Res. 2013;256:205-213. doi:10.1016/j.bbr.2013.08.026

38. Grizzell JA, Echeverria V. New Insights into the Mechanisms of Action of Cotinine and its Distinctive Effects from Nicotine. Neurochem Res. 2014;40(10). doi:10.1007/s11064- 
602

603

604

605

606

607

608

609

610

611

612

613

614

615

616

617

618

619

620

621

622

623

624

014-1359-2

39. Li CSR, Huang C, Constable RT, Sinha R. Imaging response inhibition in a stop-signal task: Neural correlates independent of signal monitoring and post-response processing. $J$ Neurosci. 2006;26(1):186-192. doi:10.1523/JNEUROSCI.3741-05.2006

40. Hampshire A, Chamberlain SR, Monti MM, Duncan J, Owen AM. The role of the right inferior frontal gyrus: inhibition and attentional control. Neuroimage. 2010;50(3):13131319. doi:10.1016/j.neuroimage.2009.12.109

41. Albert J, López-Martín S, Tapia M, Montoya D, Carretié L. The role of the anterior cingulate cortex in emotional response inhibition. Hum Brain Mapp. 2012;33(9):21472160. doi:10.1002/hbm. 21347

42. Mies GW, van der Molen MW, Smits M, Hengeveld MW, Van der Veen FM. The anterior cingulate cortex responds differently to the validity and valence of feedback in a timeestimation task. Neuroimage. 2011;56(4):2321-2328. doi:10.1016/j.neuroimage.2011.04.015

43. Luft CDB. Learning from feedback: The neural mechanisms of feedback processing facilitating better performance. Behav Brain Res. 2014;261:356-368.

doi:10.1016/j.bbr.2013.12.043

44. Galván A, Poldrack RA, Baker CM, McGlennen KM, London ED. Neural correlates of response inhibition and cigarette smoking in late adolescence. Neuropsychopharmacology. 2011;36(5):970-978. doi:10.1038/npp.2010.235

45. Chao HHA, Luo X, Chang JLK, Li CSR. Activation of the pre-supplementary motor area but not inferior prefrontal cortex in association with short stop signal reaction time--an intra-subject analysis. BMC Neurosci. 2009;10(1):1-10. doi:10.1186/1471-2202-10-75 
bioRxiv preprint doi: https://doi.org/10.1101/2021.12.08.471840; this version posted December 10,2021 . The copyright holder for this preprint

(which was not certified by peer review) is the author/funder, who has granted bioRxiv a license to display the preprint in perpetuity. It is made available under aCC-BY-NC-ND 4.0 International license.

625 46. Li CSR, Luo X, Yan P, Bergquist K, Sinha R. Altered impulse control in alcohol

626 dependence: Neural measures of stop signal performance. Alcohol Clin Exp Res.

627 2009;33(4):740-750. doi:10.1111/j.1530-0277.2008.00891.x

628

629

630

631

632

633

634

635

636

637

638

639

640

641

642 Figure Captions 


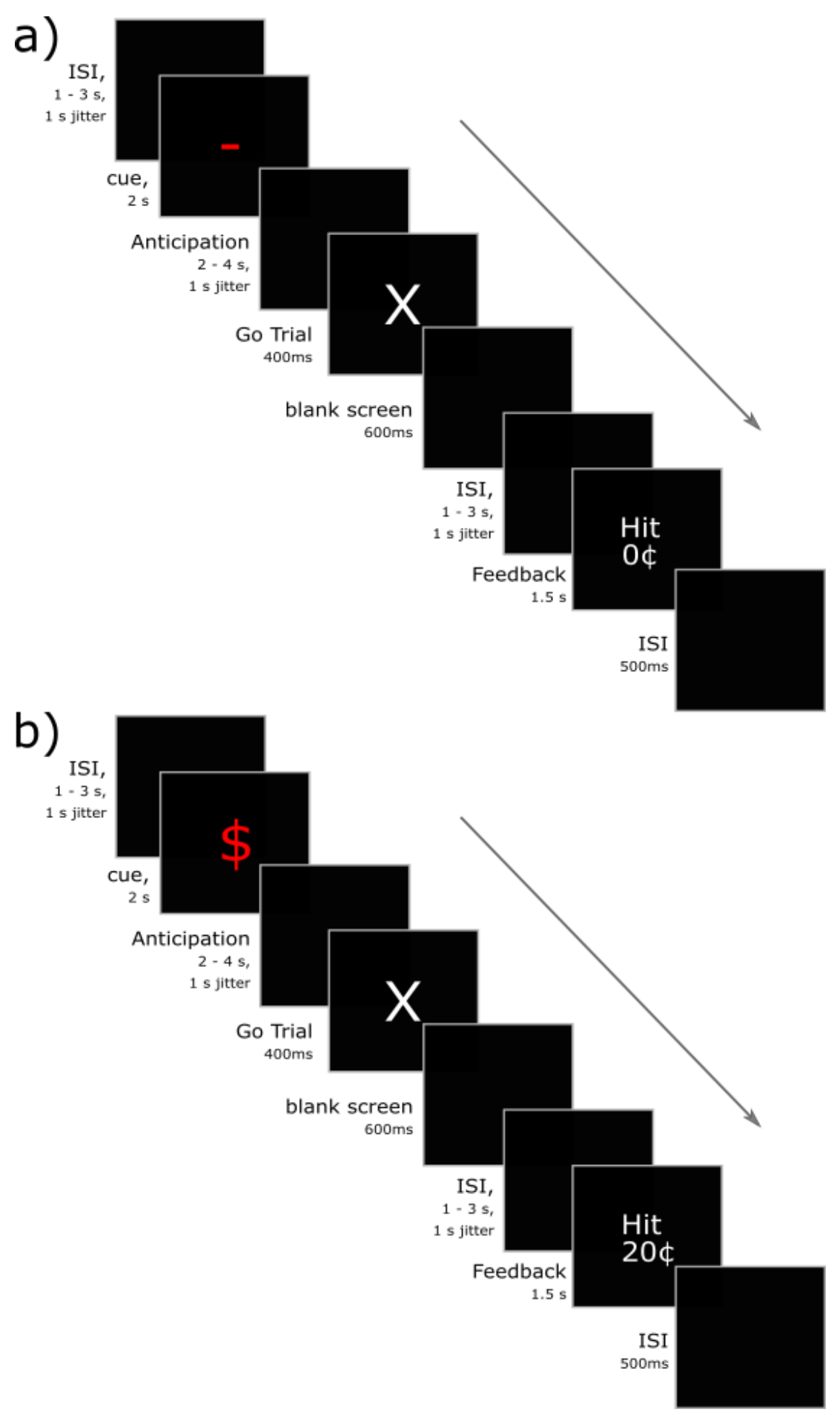

644 Figure 1. Example sequences of go-trials. If the cue symbol was a dash ("-“), the upcoming trial was a 645 neutral trial and no money could be won, irrespective of performance. If it was a dollar symbol ("\$"), the upcoming trial would be a reward trial where monetary rewards could be won, depending on performance. If the color of the symbol was white - there was a $20 \%$ probability that the upcoming trial would be a stop trial. If this color was red - it would be $40 \%$ probability. a) N40 go-trial which is accurately performed (correct button-press under 400ms). Feedback is "hit" but because this is a neutral trial, no monetary reward 

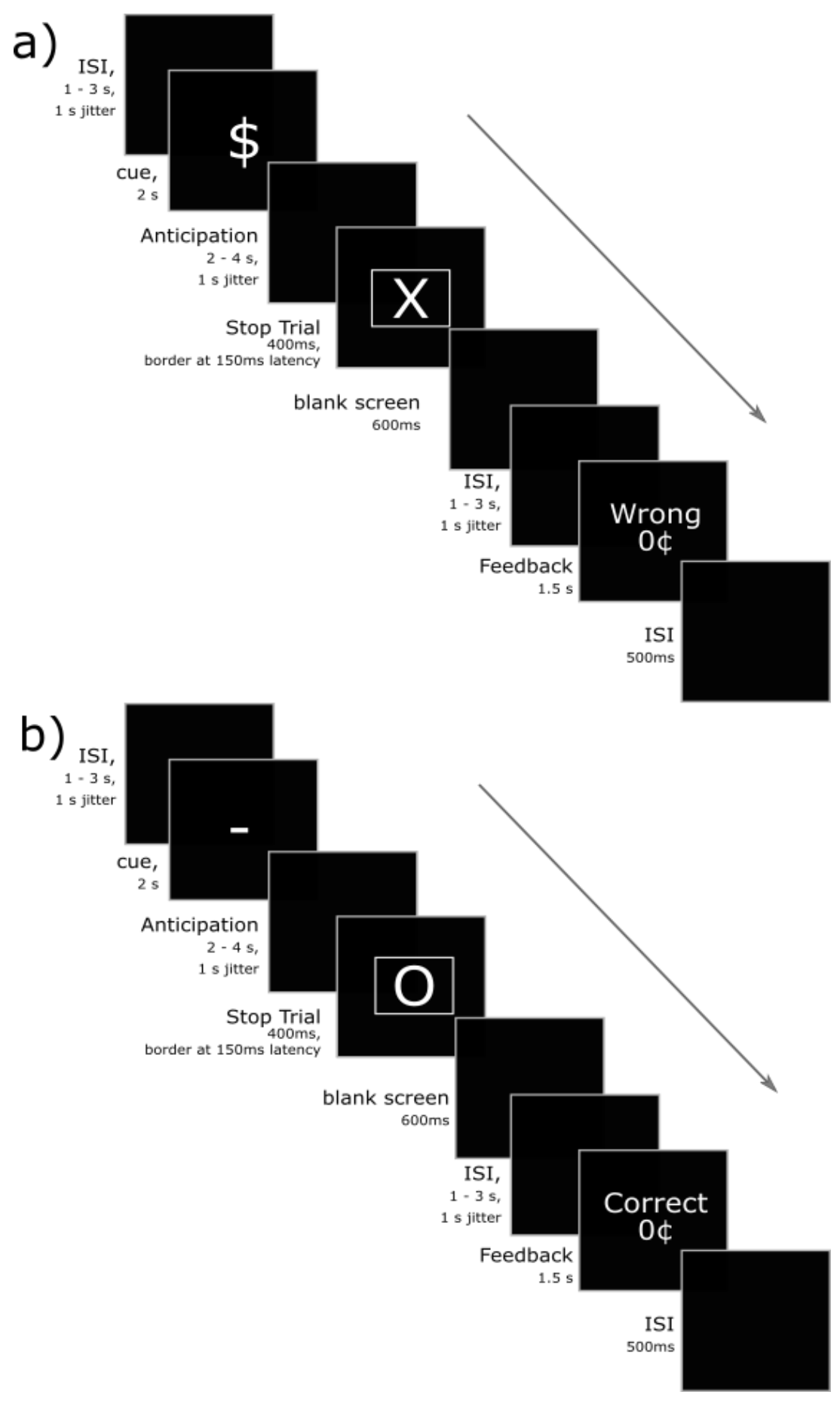

654 Figure 2. Example sequences of stop-trials. If the cue symbol was a dash (““-"), the upcoming trial was a neutral trial and no money could be won, irrespective of performance. If it was a dollar symbol ("\$"), the upcoming trial would be a reward trial where monetary rewards could be won, depending on performance. If the color of the symbol was white - there was a $20 \%$ probability that the upcoming trial would be a stop 
trial. If this color was red - it would be $40 \%$ probability. a) R20 stop-trial with the stop signal appearing after 150ms latency. In this example, the participant failed to inhibit a response and did not respond within the $400 \mathrm{~ms}$. Feedback "wrong" is given for responding (failed inhibition) and no reward is given as the response wasn't under 400ms. b) N20 stop-trial with the stop signal appearing after 150ms latency. In this example, the participant accurately inhibited their response (successful inhibition).

a)

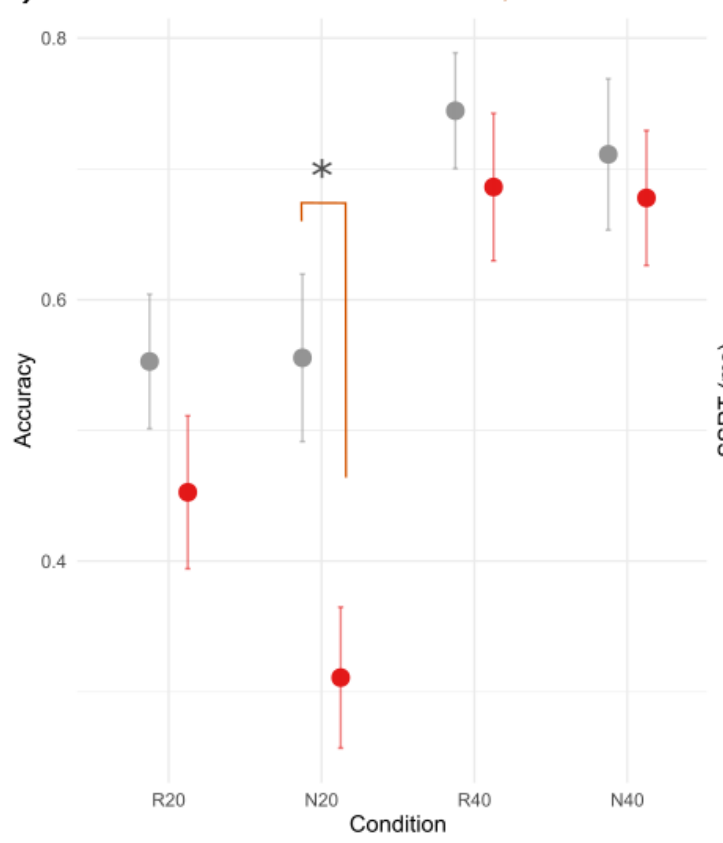

b) Stop Signal Reaction Time

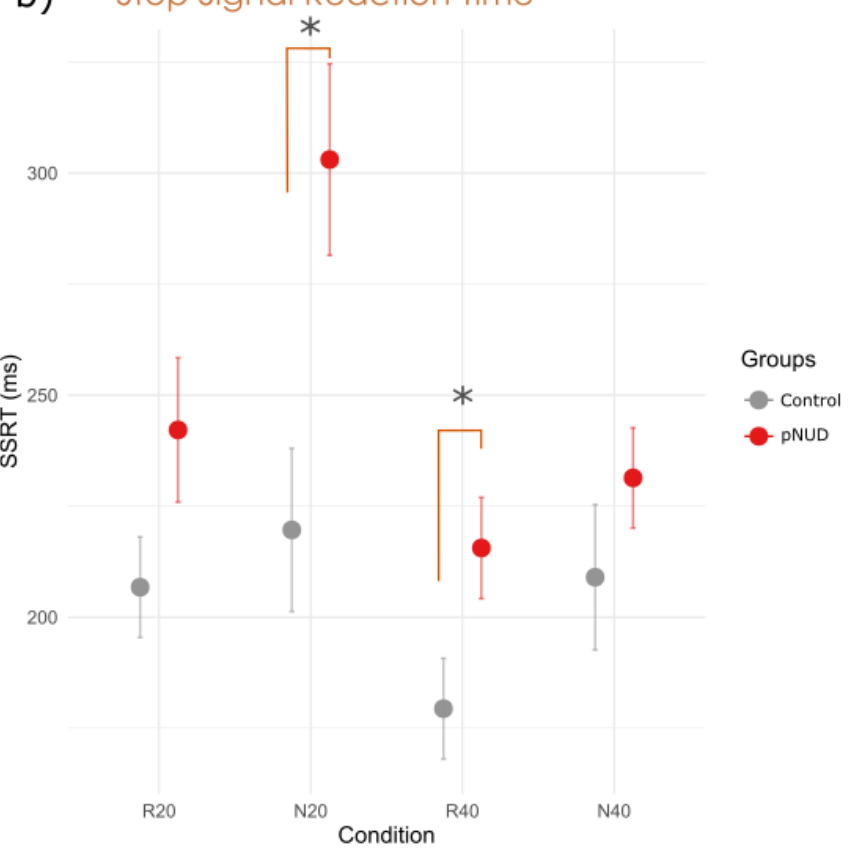

Figure 3. Inhibition accuracy measures for the people with a nicotine use disorder (pNUD) and control groups. a) Stop trial accuracy scores. Both groups show similar performance here, with no main effect group $\mathrm{F}(1,38)=3.2, \mathrm{p}=0.08, \eta_{\mathrm{p}}{ }^{2}=0.078$. There is a main effect of probability $\mathrm{F}(1,38)=65.95, \mathrm{p}<0.0001 *$, $\eta_{\mathrm{p}}{ }^{2}=0.63$, and group x probability interaction $\mathrm{F}(1,38)=4.70 \mathrm{p}=0.036^{*}, \eta_{\mathrm{p}}{ }^{2}=0.11$. There was a significant difference between N20 condition for control and pNUD group (t-test, $\mathrm{p}<0.01$ ). There were no significant main effect of reward $\left(\mathrm{F}(1,38)=2.3, \mathrm{p}=0.14, \eta_{\mathrm{p}}{ }^{2}=0.058\right)$, no group and reward interaction $(\mathrm{F}(1,38)=1.3$, $\left.\mathrm{p}=0.1, \eta_{\mathrm{p}}{ }^{2}=0.026\right)$ and no three way group, probability and reward interaction $(\mathrm{F}(1,38)=2.98, \mathrm{p}=0.09$, 0.073 ). b) The pNUD group have slower SSRTs (main effect of group: $F(1,37)=8.09, p<0.01, \eta_{p}{ }^{2}=0.18$ ). There is a significant main effect of reward $\left(\mathrm{F}(1,37)=12.96, \mathrm{p}<0.001, \eta_{\mathrm{p}}{ }^{2}=0.26\right) *$ and probability $(\mathrm{F}(1,37)$ $\left.=13.13, \mathrm{p}<0.001, \eta_{\mathrm{p}}{ }^{2}=0.26\right)^{*}$. There is a trend of a three way interaction between group, probability and reward $\left(\mathrm{F}(1,37)=3.92, \mathrm{p}=0.055, \eta_{\mathrm{p}}^{2}=0.096\right)$. There were no significant two way interactions between group and reward $\left(\mathrm{F}(1,37)=1.1, \mathrm{p}=0.3, \eta_{\mathrm{p}}{ }^{2}=0.03\right)$, reward and probability $\left(\mathrm{F}(1,37)=0.8, \mathrm{p}=3.7, \eta_{\mathrm{p}}{ }^{2}=\right.$ $0.02)$ and group and probability $\left(\mathrm{F}(1,37)=2.57, \mathrm{p}=0.12, \eta_{\mathrm{p}}{ }^{2}=0.065\right) . *=p<0.05$. pNUD $=$ nicotine use disorder. 

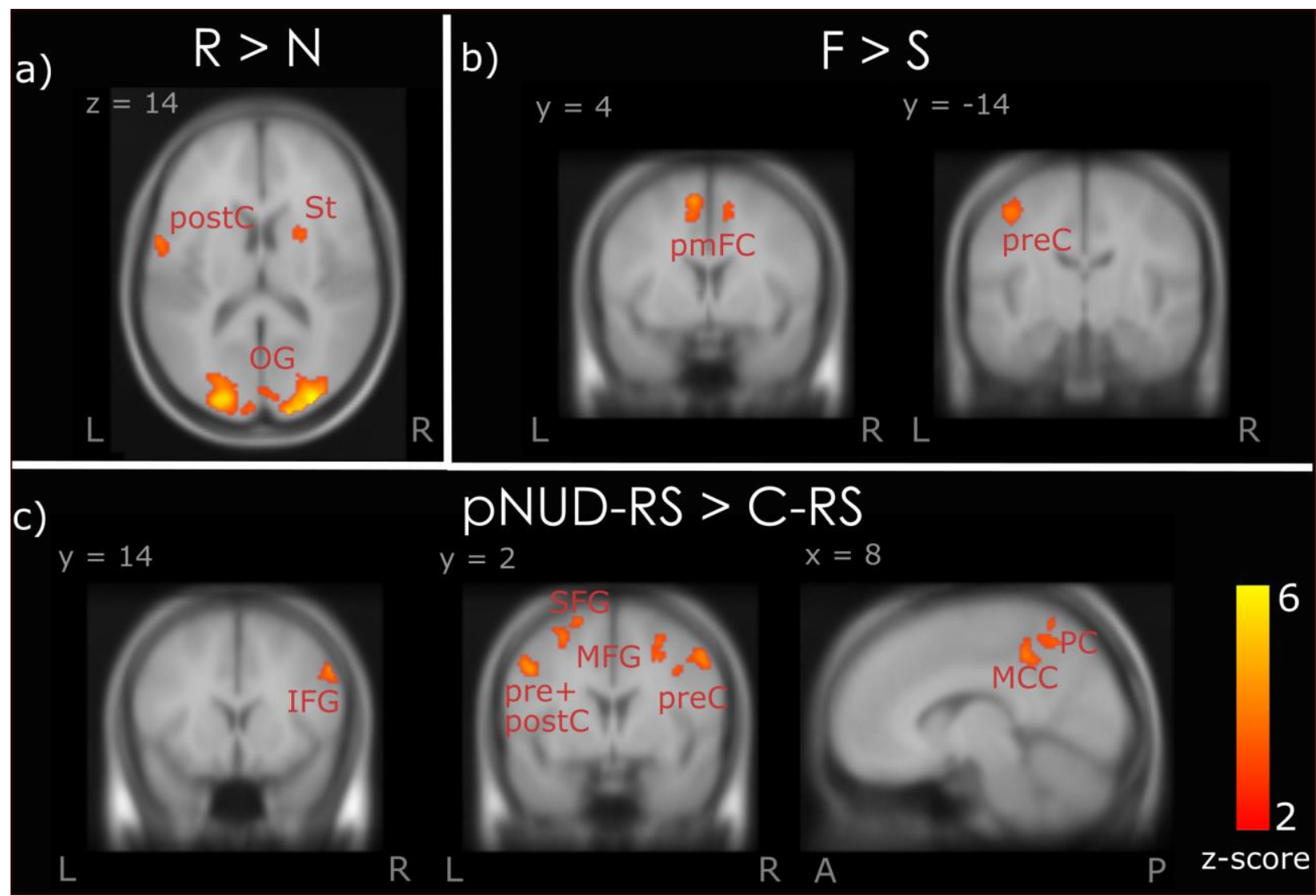

Figure 4. Activity during cue epoch. This is activity when participants are cued on the probability of an upcoming stop trial and whether it is a reward or a neutral trial. Abbreviations: $R=$ reward, $N=$ neutral, $F$ $=$ Failed inhibition, $S=$ successful inhibition, $p N U D=$ people with a nicotine disorder group, $C=$ control group. $P C=$ precuneus,$S t=$ striatum, $p m F C=$ posterior medial frontal cortex, IFG $=$ inferior frontal gyrus, $M F G=$ middle frontal gyrus, $S F G=$ superior frontal gyrus, preC $=$ precentral, post $C=$ postcentral, $M C C=$ mid cingulate cortex,$O G=$ occipital gyrus. $L=$ left, $R=$ Right. $A=$ anterior, $P=$ posterior. $* M F G$ + SFG activity in Figure 3c is pFWE $=0.055$ (cluster-level). 

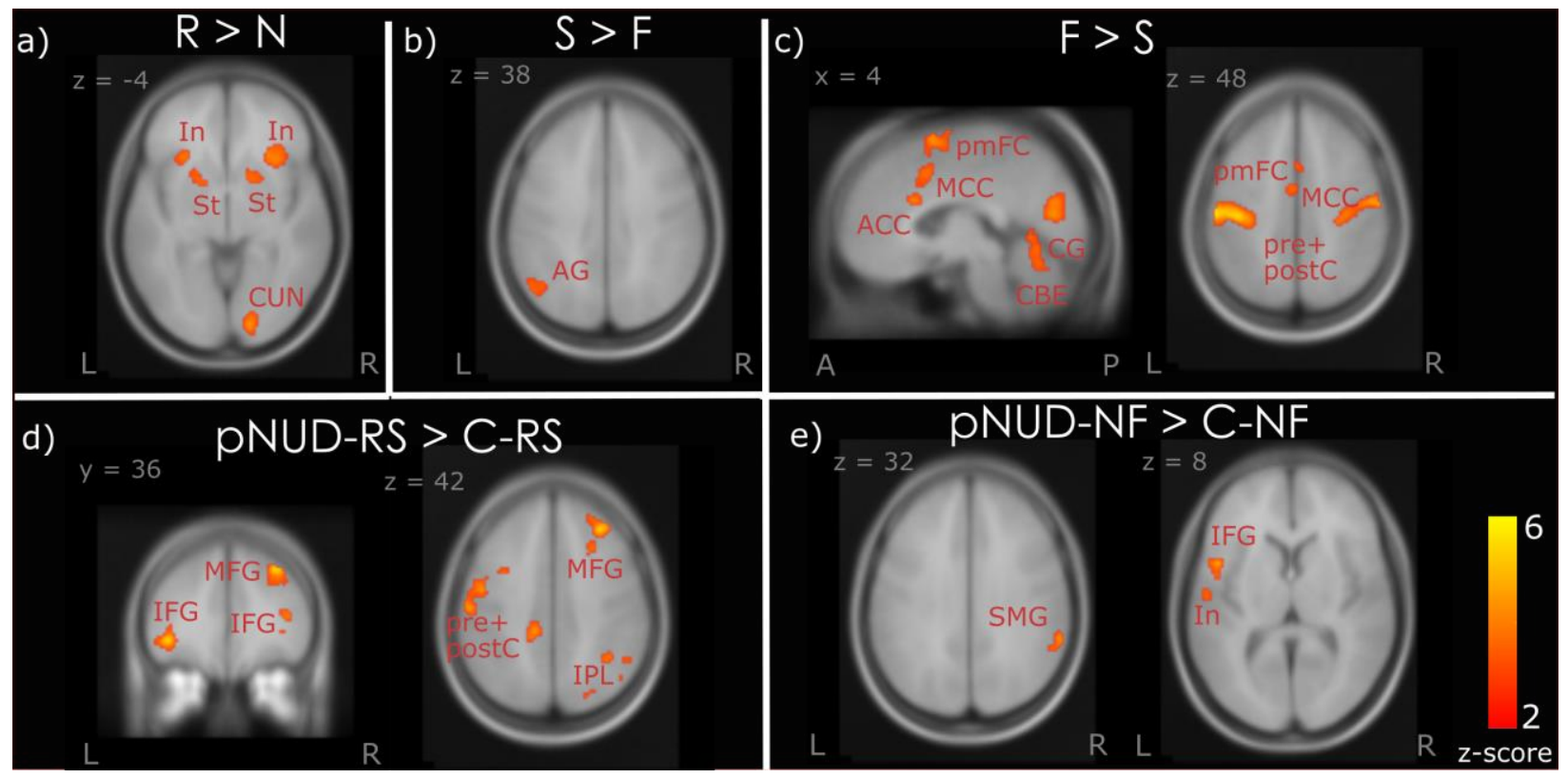

Figure 5. Brain activity during the anticipation epoch. This is activity immediately prior to the onset of the trial. Abbreviations: $R=$ reward, $N=$ neutral, $F=$ Failed inhibition, $S=$ successful inhibition, $p N U D$ $=$ people with a nicotine disorder group, $C=$ control group, $R S=$ reward successful inhibition trials, $N F$ $=$ neutral failed inhibition trials $I n=$ insula, $S t=$ striatum, $C U N=$ cuneus, $A C C=$ anterior cingulate cortex, $M C C=$ midcingulate cortex,$p m F C=$ posterior medial frontal cortex, pre $C=$ precentral, post $C=$ postcentral, $I F G=$ inferior frontal cortex, $S M G=$ supramarginal gyrus, $M F G=$ middle frontal cortex, IPL
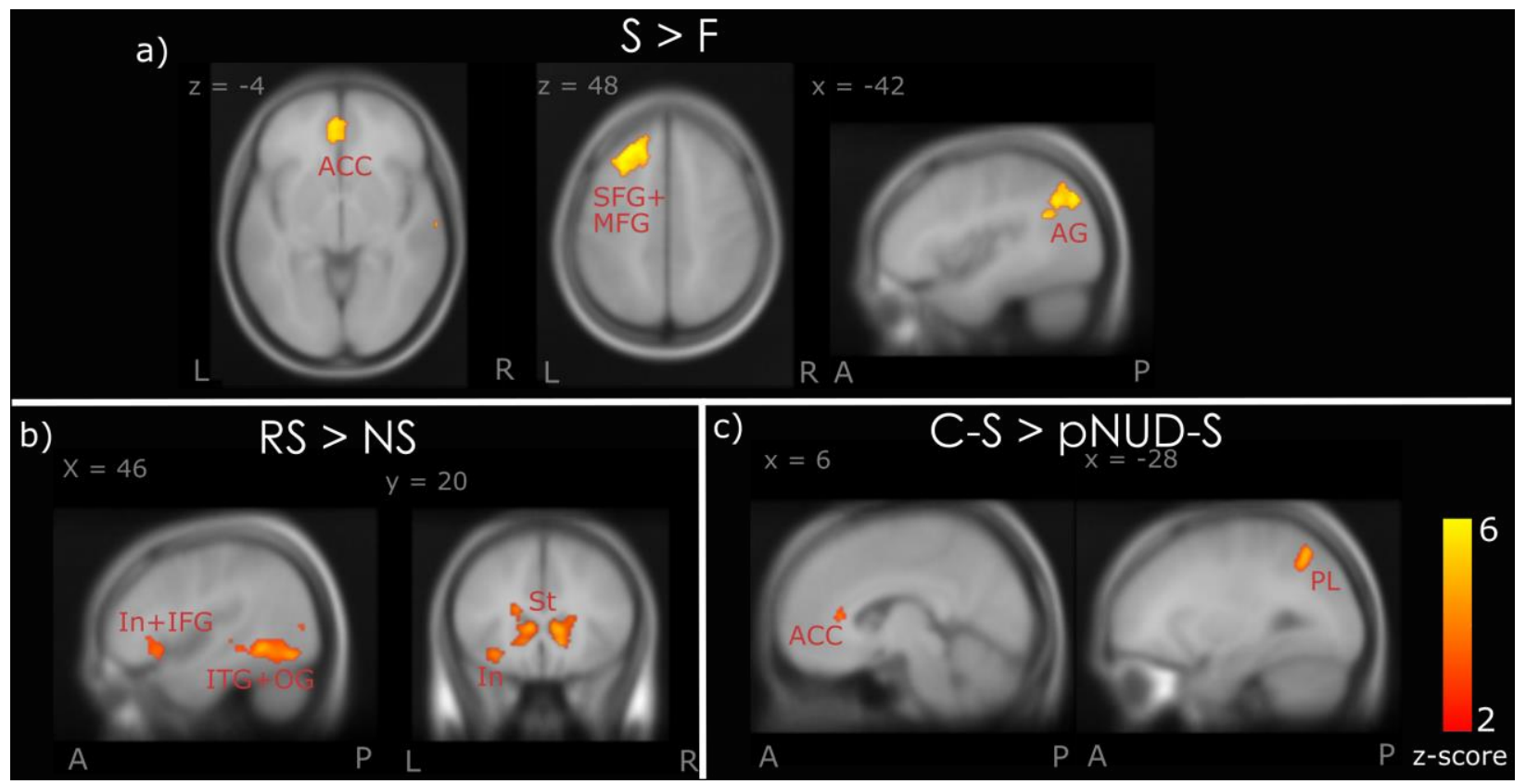

Figure 6. Brain activity at the stop-trial epoch. This is activity at the trial - when the stop signal is presented, and participants need to inhibit a response to make progress towards the larger later reward. Abbreviations: $R=$ reward, $N=$ neutral, $F=$ Failed inhibition, $S=$ successful inhibition, $R S=$ reward 
bioRxiv preprint doi: https://doi.org/10.1101/2021.12.08.471840; this version posted December 10, 2021. The copyright holder for this preprint (which was not certified by peer review) is the author/funder, who has granted bioRxiv a license to display the preprint in perpetuity. It is made available under aCC-BY-NC-ND 4.0 International license.

successful inhibition trials, NS = neutral successful inhibition trials, pNUD = people with a nicotine disorder group, $C=$ control group. ACC = anterior cingulate cortex, $S F G=$ superior frontal gyrus, $M F G$ $=$ middle frontal gyrus, In = insula, IFG = inferior frontal gyrus, $S t=$ striatum, $A G=$ angular gyrus, $P L$ = parietal lobule. $L=$ left, $R=$ Right. $A=$ anterior, $P=$ posterior. ${ }^{*}$ The successful $>$ failed inhibitions contrast here used threshold of $p<0.05$ FWE corrected at whole brain level
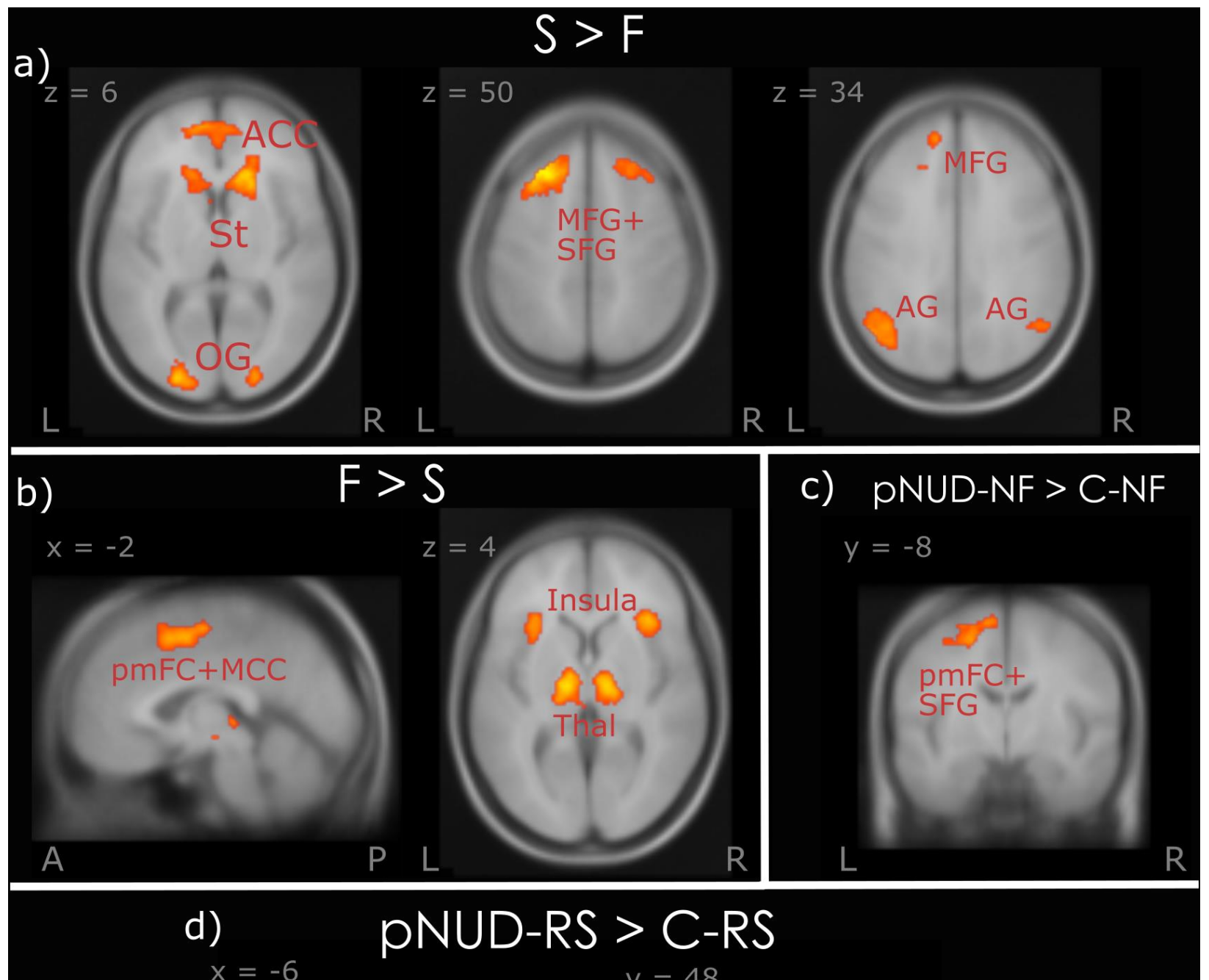

$x=-6$

$$
y=48
$$
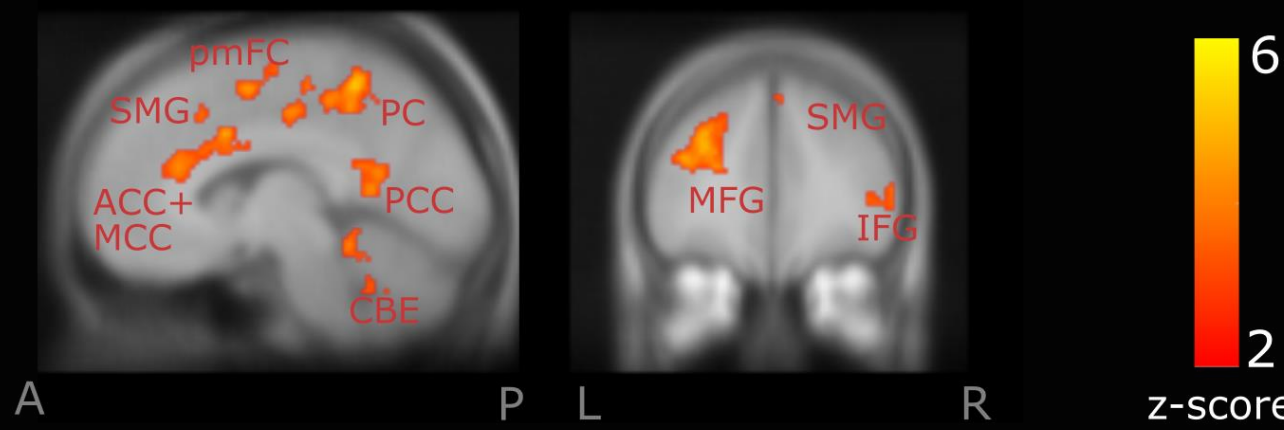

P L

$\mathrm{R}$

z-score 
Figure 7. Brain activity at the feedback epoch. This is activity at the feedback epoch, where participants are given feedback of "correct $0 \mathrm{c}$ " for successful inhibitions and "incorrect $0 \mathrm{c}$ " for failed inhibitions, where the response was slower than $400 \mathrm{~ms}$ or was the incorrect button press. Abbreviations: $R=$ reward, $N=$ neutral, $F=$ Failed inhibition, $S=$ successful inhibition, $R S=$ reward successful inhibition trials, $N S=$ neutral successful inhibition trials, pNUD = people with a nicotine disorder group, $C=$ control group. $A C C=$ anterior cingulate cortex, $S F G=$ superior frontal gyrus, $M F G=$ middle frontal gyrus, In = insula, $I F G=$ inferior frontal gyrus, $M C C=$ midcingulate gyrus, $P C C=$ posterior cingulate cortex, pmFC $=$ posterior medial frontal cortex, $O G=$ occipital gyrus, $S t=$ striatum, Thal = thalamus, $C B E=$ cerebellum, $S M G=$ superior medial gyrus, $A G=$ angular gyrus. $L=$ left, $R=$ Right. $A=$ anterior, $P=$ posterior . 


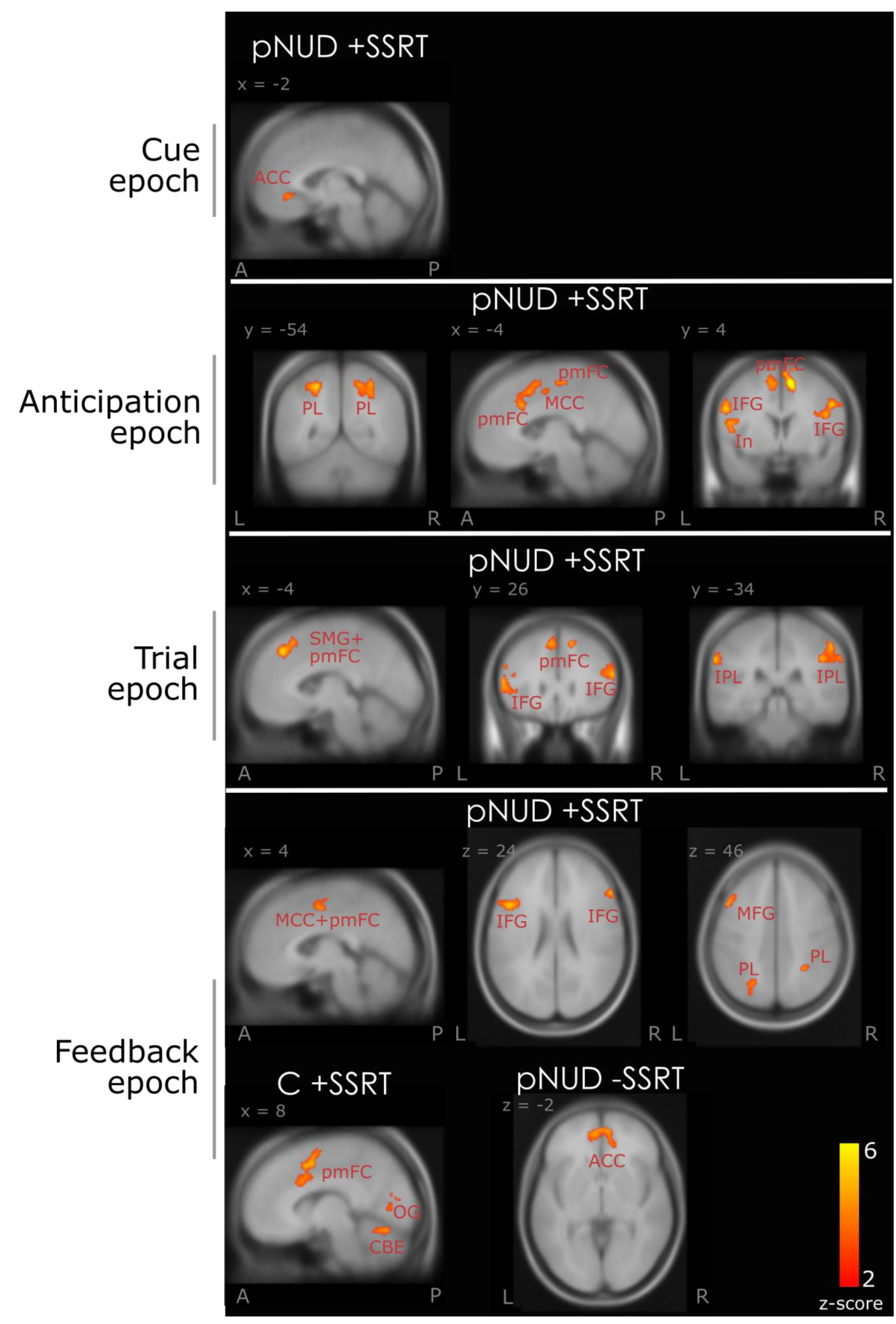


Figure 8. Brain activity correlating with SSRTs. pNUD+ SSRT = slower (increasing) SSRT correlating with the people with a nicotine disorder group. D- SSRT = faster (decreasing) SSRT correlating with the people with a nicotine disorder group. C+ SSRT $=$ slower (increasing) SSRT correlating with the control group. Abbreviations: $A C C=$ anterior cingulate cortex, $M C C=$ midcingulate cortex, pmFC $=$ posterior medial frontal cortex, IFG = inferior frontal gyrus, $S F G=$ superior frontal gyrus, $M F G=$ middle frontal 